\title{
Room Temperature Storage of Methane by Bamboo-Like Carbon Nanotubes
}

\author{
Mitsunori Furuya, Kenji Ichimura \\ Graduate School of Science and Technology, Kumamoto University, Kumamoto, Japan \\ Email: ichimura@kumamoto-u.ac.jp
}

Received May 9, 2013; revised June 9, 2013; accepted July 9, 2013

Copyright (C) 2013 Mitsunori Furuya, Kenji Ichimura. This is an open access article distributed under the Creative Commons Attribution License, which permits unrestricted use, distribution, and reproduction in any medium, provided the original work is properly cited.

\begin{abstract}
Single carbon nanotubes (CNTs) have the sorption states at around $200 \mathrm{~K}$, and show the poor desorption characteristics at around $300 \mathrm{~K}$. The bamboo-like CNTs, HP1050 and the ground HP1050 (HP-1050G), show the desorption peaks at around $100 \mathrm{~K}$ and $140 \mathrm{~K}$, respectively. Furthermore, HP1050G has the sorption states at around $300-400 \mathrm{~K}$. On the basis of the sorption process, the ground bamboo-like CNT has a possibility for application to the room temperature storage of methane, which has utility for energy technologies such as fuel cells.
\end{abstract}

Keywords: Methane; Storage; Carbon Nanotube; Absorption States

\section{Introduction}

Methan storage characteristics in porous metal-organic frameworks have been reported [1].

We have reported the chemical interactions or storage of hydrogen and of rare gases, such as $\mathrm{He}, \mathrm{Ne}$, and $\mathrm{Ar}$, in solid carbon nanotubes [2]. Closed carbon nanotubes (CNTs) show larger amounts of absorption for gases such as hydrogen, $\mathrm{He}, \mathrm{Ne}$, and Ar than opened CNTs. From these results, we conclude that sites preferentially found in endcaps are more active for the chemical interaction between rare gases and solid carbon nanotubes. Recently, bamboo-like CNTs that have many endcap structures have been produced in large quantity [3]. According to the above-mentioned results, these kinds of CNTs are expected to have strong interactions for gases.

On the basis of the sorption characteristics for CNTs, we examined the thermal desorption of methane from CNTs or the sorption abilities of CNTs for methane.

\section{Experimental}

Capped and open (no endcaps) single wall carbon nanotubes (CSWCNT and OSWCNT, Bucky USA BU-202 (endcaps) and BU-203 (no endcaps), respectively, with $1.4-3 \mathrm{~nm}$ diameter and $10-50 \mu \mathrm{m}$ length) were used without further purification for the adsorption studies. The only difference between BU-202 and BU-203 is the endcap structure at both ends, and otherwise the two structures are the same.
The synthesis of CNT with a high density of bamboo structures, HP1050, was carried out in a quartz-tube reactor [3]. Figure 1 shows the TEM image of sample HP1050. For each synthesis run, $100 \mathrm{mg}$ of the catalyst powder was spread on to a Mo boat $\left(40 \times 100 \mathrm{~mm}^{2}\right)$. Prior to rising the temperature, the reactor chamber was flushed with $\mathrm{N}_{2}$ flowing at $100 \mathrm{sccm}$ for 20 minutes. Then the growth of nanotubes was performed by flowing gas mixtures of $\mathrm{N}_{2}(100 \mathrm{sccm}), \mathrm{NH}_{3}(40 \mathrm{sccm})$ and $\mathrm{C}_{2} \mathrm{H}_{2}$ $(10 \mathrm{sccm})$ at $1050^{\circ} \mathrm{C}$ and 760 Torr for $2 \mathrm{~h}$. Sample HP1050G was obtained from HP1050 using a ball mill at room temperature under ambient conditions. The TEM image of HP1050G was almost the same as Figure 1. The specific surface area was measured by means of the $\mathrm{N}_{2}$ BET method at $77 \mathrm{~K}$.

After vacuum heating at $653 \mathrm{~K}$ or $1073 \mathrm{~K}$, the samples were exposed to methane (Nippon Sanso, $>99.9 \%$ purity) of 1 to $1.4 \mathrm{~atm}$, at $473 \mathrm{~K}$ for 3 - 5 days. After the sample was cooled to liquid nitrogen temperature, the sample tube was evacuated to ultra-high vacuum. Desorbed gases were analyzed by using two mass-spectrometers when the sample was heated at a temperature-rise rate of $5 \mathrm{~K} / \mathrm{min}$.

\section{Results and Discussion}

Figure 2 shows the thermal desorption of methane from CNTs. Methane desorption is confirmed from the fragmentation in the mass number regions $1-2$ and $12-16$. 


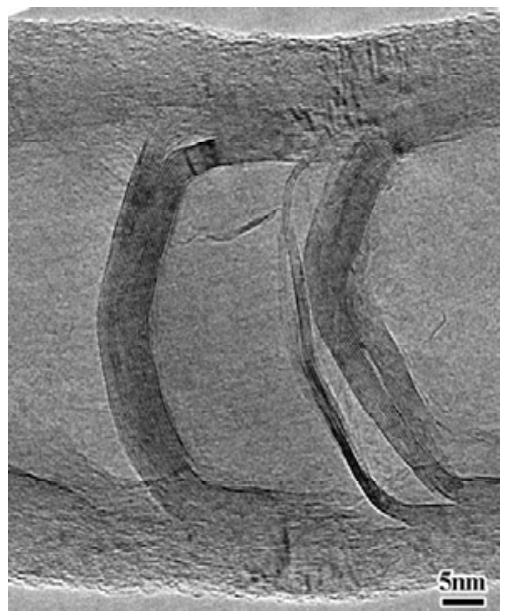

Figure 1. TEM image of HP1050.

No significant signals of hydrogen and hydrocarbon are detected. In order to avoid an influence of $\mathrm{O}^{+}$from $\mathrm{H}_{2} \mathrm{O}$, methane desorption is recorded by monitoring the mass number of 15 .

Among the various CNTs, SWCNTs show relatively strong interactions for hydrogen [2]. From Figure 2, the desorption of methane by a van der Waals interaction is observed at around $100 \mathrm{~K}$. Additional desorption peaks appear at around $180 \mathrm{~K}$ and $220 \mathrm{~K}$ for the closed and open SWCNTs, respectively. Since the desorption occurs at higher temperatures than the boiling point of methane, 111.7 K, this desorption is considered to be due to an extended van der Waals interaction between ad-/ab-sorbed methane and CNT. A broad desorption, due to chemical interaction/bonding, was also observed at around $900 \mathrm{~K}$ and $1000 \mathrm{~K}$, respectively, for the closed and open SWCNTs. The ad-/ab-sorption characteristics at around $180-220 \mathrm{~K}$ and $900-1000 \mathrm{~K}$ for SWCNTs are, respectively, useful for low temperature storage and gettering (vacuum-pumping) applications.

The bamboo-like CNT, HP1050 showed a clean thermal desorption peak for methane at $95 \mathrm{~K}$, due to the van der Waals interaction. Although thermal desorption is a kinetic measurement method and the ad-/ab-sorption amount at equilibrium cannot be determined, thermal desorption gives important information about the ad-/ab-sorption states, and at least the amount of ad-/ab-sorption remaining under ultra-high vacuum conditions can be evaluated. As shown in the inset to Figure 2, the bamboo-like CNTs show desorption of methane in the temperature range $220-650 \mathrm{~K}$, and $\mathrm{HP} 1050 \mathrm{G}$ shows a stronger effect than HP1050. A stronger extended van der Waals interaction is realized in bamboo-like CNTs. The high temperature desorption observed for the SWCNTs almost disappears for the bamboo-like CNTs.

On the other hand, the ground bamboo-like CNTs, HP1050G, show a thermal desorption peak at $144 \mathrm{~K}$.

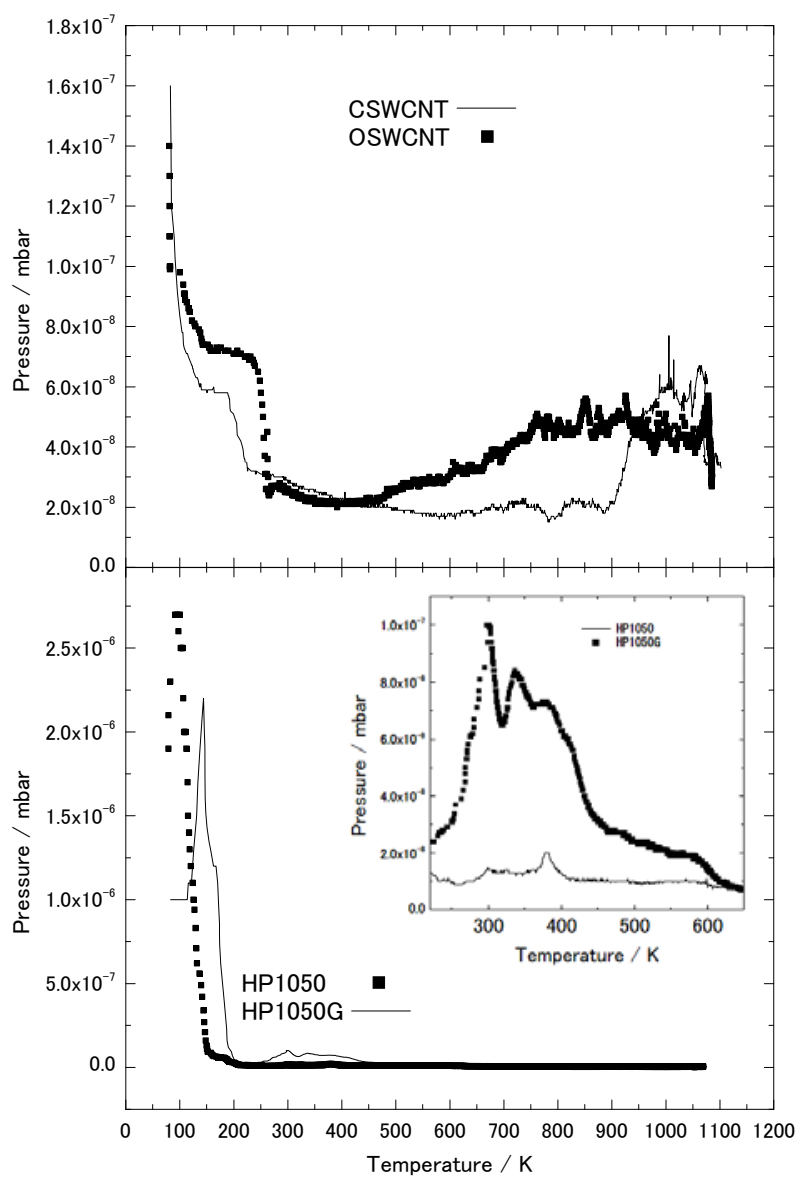

Figure 2. Thermal desorption of methane from SWCNTs and bamboo-like CNTs. The inset show the desorption in the temperature range $220-650 \mathrm{~K}$.

Since the peak temperature is higher than the boiling point of methane, the mechanism for this desorption should be assigned to the extended van der Waals interaction. The grinding treatment causes a shortening of the tube as observed in TEM images and causes the specific surface area to increase from $21 \mathrm{~m}^{2} / \mathrm{g}$ to $245 \mathrm{~m}^{2} / \mathrm{g}$. The grinding treatment changes the properties of the bamboo-like CNT, and presumably exposes more bamboo cap surfaces to the methane gas. Active sites having new electronic states and nano-space are considered to be generated in the bamboo joints, wall-cap joints, as seen in Figure 1.

Furthermore, as shown in the inset figure, new absorption states were observed at around $350 \mathrm{~K}$ for HP1050G. The reason for this methane absorption is considered to be due to a super van der Waals interaction or weak chemical bonding between the ad-/ab-sorbed methane and the CNT. The high temperature desorption observed for the SWCNTs also disappears for the bamboo type tubes.

In thermal desorption method, the amount of desorption was calculated by using the pumping rate, the meas- 
Table 1. Amounts of desorption of Methane (mol) per gram CNTs in nominal three temperature regions: low, medium, and high temperatures.

\begin{tabular}{cccc}
\hline Sample & $\mathrm{T}<250 \mathrm{~K}$ & $250 \mathrm{~K}, \mathrm{~T}<450 \mathrm{~K}$ & $450 \mathrm{~K}<\mathrm{T}$ \\
\hline CSWCNT & 0.2 & 0.03 & 1.8 \\
OSWCNT & 0.2 & 0.06 & 3.1 \\
HP1050 & 6.6 & 0.02 & 0 \\
HP1050G & 11.3 & 1.1 & 0 \\
\hline
\end{tabular}

Units: $\times 10^{-5} \mathrm{~mol} \mathrm{CH}_{4} / \mathrm{g} \mathrm{CNTs}$.

urement volume and the sensitivity. The amount of desorption means a remained amount under the high-vacuum measurement conditions. However, this suggests the comparison for the sorption characteristics among CNTs. The amounts of desorbed methane are evaluated by using the values of the integrated desorption area, background pressure, pumping speed, system volume, and sensitivity, and the results are listed in Table 1. Since the amount of desorbed methane means the amount remaining after of ad-/absorption under high-vacuum conditions, the ad-/ab-sorption amount at equilibrium is expected to increase at high methane pressures at around room temperature. At least, room temperature storage requires ad-/ab-sorption states at around room temperature. HP1050G satisfies this requirement. HP1050G shows a larger amount of desorption than HP1050, the active sites are assigned not to the tube or bundle structure but rather to the endcap-like structure of the joint or gnarl of the bamboo feature. Noble electronic states appear in the endcap-like structure of the joint or gnarl of the bamboo feature in HP1050G.

\section{Conclusions}

The bamboo-like CNTs, HP1050 and the ground HP1050 (HP-1050G), show the desorption peaks at around $100 \mathrm{~K}$ and $140 \mathrm{~K}$, respectively. Furthermore, HP1050G has the sorption states at around $300-400 \mathrm{~K}$. By using the special characteristics of the sorption for the ground bamboo-like CNT, the room temperature storage of methane can be realized.

\section{Acknowledgements}

Authors thank Dr. W. Z. Li and Associate Professor Z. F. Ren of Department of Physics, Boston College for the sample supply of the bamboo-like CNTs.

\section{REFERENCES}

[1] W. Zhou, "Methane Storage in Porous Metal-Organic Frameworks: Current Records and Future Perspectives," The Chemical Record, Vol. 10, No. 3, 2010, pp. 200-204. doi:10.1002/tcr.201000004

[2] K. Ichimura, K. Imaeda, C.-W. Jin and H. Inokuchi, "Super van der Waals Interaction of Fullerenes and Carbon Nanotubes with Rare Gases and Hydrogen-Storage Characteristics," Physica B: Condensed Matter, Vol. 323, No. 1-4, 2002, pp.137- 139. doi:10.1016/S0921-4526(02)00879-7

[3] W. Z. Li, J. G. Wen and Z. F. Ren, "Effect of Temperature on Growth and Structure of Carbon Nanotubes by Chemical Vapor Deposition," Applied Physics A, Vol. 74, No. 74, 2002, pp. 397-402. doi: $10.1007 / \mathrm{s} 003390201284$ 